\title{
Probing the transformation of discontinuous associations into episodic memory: An event-related fMRI study
}

\author{
Shaozheng Qin, ${ }^{\mathrm{a}, \mathrm{b}, \mathrm{c}}$ Carinne Piekema, ${ }^{\mathrm{b}, \mathrm{d}}$ Karl Magnus Petersson, ${ }^{\mathrm{b}}$ Buxin Han, ${ }^{\mathrm{a}}$ \\ Jing Luo, ${ }^{\mathrm{a}, *}$ and Guillén Fernández ${ }^{\mathrm{b}, \mathrm{e}, *}$ \\ ${ }^{a}$ Key Laboratory of Mental Health, Institute of Psychology, Chinese Academy of Sciences (CAS), 100101 Beijing, China \\ ${ }^{\mathrm{b}}$ F.C. Donders Centre for Cognitive Neuroimaging, Radboud University Nijmegen, 6500 HB Nijmegen, The Netherlands \\ ${ }^{\mathrm{c}}$ Graduate School, Chinese Academy of Sciences (CAS), 100039 Beijing, China \\ ${ }^{\mathrm{d}}$ Helmholtz Institute, Utrecht University, 3584 CS Utrecht, The Netherlands \\ ' Department of Neurology, University Medical Center Nijmegen, 6500 HB Nijmegen, The Netherlands
}

Received 13 April 2007; revised 12 June 2007; accepted 12 July 2007

Available online 3 August 2007

\begin{abstract}
Using event-related functional magnetic resonance imaging, we identified brain regions involved in storing associations of events discontinuous in time into long-term memory. Participants were scanned while memorizing item-triplets including simultaneous and discontinuous associations. Subsequent memory tests showed that participants remembered both types of associations equally well. First, by constructing the contrast between the subsequent memory effects for discontinuous associations and simultaneous associations, we identified the left posterior parahippocampal region, dorsolateral prefrontal cortex, the basal ganglia, posterior midline structures, and the middle temporal gyrus as being specifically involved in transforming discontinuous associations into episodic memory. Second, we replicated that the prefrontal cortex and the medial temporal lobe (MTL) especially the hippocampus are involved in associative memory formation in general. Our findings provide evidence for distinct neural operation(s) that supports the binding and storing discontinuous associations in memory. We suggest that top-down signals from the prefrontal cortex and MTL may trigger reactivation of internal representation in posterior midline structures of the first event, thus allowing it to be associated with the second event. The dorsolateral prefrontal cortex together with basal ganglia may support this encoding operation by executive and binding processes within working memory, and the posterior parahippocampal region may play a role in binding and memory formation.
\end{abstract}

(C) 2007 Elsevier Inc. All rights reserved.

Keywords: Episodic memory; Hippocampus; Medial temporal lobe; Prefrontal cortex; Discontinuous association; Simultaneous association

\footnotetext{
* Corresponding authors. J. Luo is to be contacted at Key Laboratory of Mental Health, Institute of Psychology, Chinese Academy of Sciences, CAS., 100101 Beijing, China. Fax: +86 0106485 1107. G. Fernández, F.C. Donders Centre for Cognitive Neuroimaging, Radboud University of Nijmegen, PO Box 9101, 6500 HB Nijmegen, The Netherlands. Fax: +31 243610989.

E-mail addresses: luoj@psych.ac.cn (J. Luo),

guillen.fernandez@fcdonders.ru.nl (G. Fernández).

Available online on ScienceDirect (www.sciencedirect.com).
}

\section{Introduction}

The ability to associate and remember different aspects of an experience as one coherent episode is a critical feature of episodic memory (Tulving, 1983, 2002; Eichenbaum, 2004). In particular, it is crucial to bind and remember aspects of an experience that do not overlap in time as belonging to one episode. However, little is known about the neural underpinnings that support the encoding of discontinuous associations (i.e., non-overlapping in time or across temporal associations), although the neural correlates of successful memory formation for single items (Brewer et al., 1998; Fernández et al., 1998; Wagner et al., 1998; Otten et al., 2001; Ranganath et al., 2005) and simultaneously occurring inter-item associations (Henke et al., 1999; Davachi and Wagner, 2002; Sperling et al., 2003; Jackson and Schacter, 2004; Prince et al., 2005) are well-established.

Generally speaking, the medial temporal lobe (MTL) and the prefrontal cortex play a critical role in episodic memory formation, including associative memories (Buckner et al., 1999; Fernández and Tendolkar, 2001; Paller and Wagner, 2002). Numerous neuroimaging studies have shown that different substructures of the MTL and the prefrontal cortex are more active while encoding single items that are later remembered than those forgotten (Brewer et al., 1998; Fernández et al., 1998; Wagner et al., 1998; Kirchhoff et al., 2000; Otten et al., 2001; Kao et al., 2005). Specifically, activity in the anterior MTL including the hippocampus and the inferior or medial prefrontal gyrus correlated with successful formation of associative or relational memories when two or three simultaneously presented items were bound together (Dolan and Fletcher, 1997; Henke et al., 1997, 1999; Sperling et al., 2003; Jackson and Schacter, 2004; Mitchell et al., 2004; Prince et al., 2005; Uncapher et al., 2006). The parahippocampal region was also thought to play a role in contextual associative processing, such as forming spatial and non-spatial associations (Aminoff et al., 2006). Nevertheless, there is still controversy over the specific contribution of different regions within the MTL to episodic memory 
formation, especially for associative memory (Schacter and Wagner, 1999; Davachi et al., 2003; Ranganath et al., 2004a,b; Squire et al., 2004). Moreover, in the studies mentioned above, information - either an item or an inter-item association belonging to one episodic experience - was presented simultaneously. Thus, there is no evidence for neural processes that support the storage of discontinuous associations in memory.

Several models have suggested that the hippocampus is critically engaged in bridging discontinuities across time (Rawlins, 1985; Wallenstein et al., 1998; Eichenbaum, 2004). For instance, in trace conditioning, animals with hippocampal lesions show, compared to sham operated animals, impaired formation of discontinuous events when the conditioned stimulus and the unconditioned stimulus do not overlap in time (Thompson-Schill et al., 1997). Based on the integration of results obtained in experimental animal and human behavioral work, Wallenstein and colleagues' developed a computational framework suggesting that the hippocampus plays a critical role in memory formation when discontinuous items have to be associated in terms of their temporal or spatial positioning or both (Wallenstein et al., 1998). Additionally, single-cell recordings from the frontal cortex in monkeys suggest that prefrontal neurons are essential for the temporal integration of visual and auditory stimuli that are separated in time (Fuster et al., 2000). Moreover, a recent event- related fMRI study has shown that, relative to binding word-pairs between constituents simultaneously presented, binding word-pairs between constituents across time (i.e., 'discontinuity association') engaged the left hippocampus (Luo and Niki, 2005). However, that study probed neural activity associated with the processing of discontinuous events in general, irrespectively of subsequent memory performance. Hence, it remains unclear whether there are distinct anatomical structures that specifically support the formation of discontinuous associations or whether they overlap with brain regions involved in the formation of associative memories in general.

Here we set out to identify those brain regions that are involved in storing discontinuous associations into episodic memory. Participants were scanned while memorizing item-triplets consisting of a simultaneous association and a discontinuous association (Fig. 1). Afterwards, participants performed single-item and associative recognition memory tests. The single-item recognition memory test is used to ensure whether the center-item of each triplet was remembered, whereas the associative recognition memory test is used to examine whether the associations were formed successfully. This set-up allows us to confirm neural correlates of memory formation for single items and associations. Additionally, and most critically for the question at issue, the contrast between the subsequent memory effects for discontinuous
Sudy Phase (fMRI)



Post-scan Memory Tests



Associative Recognition Memory
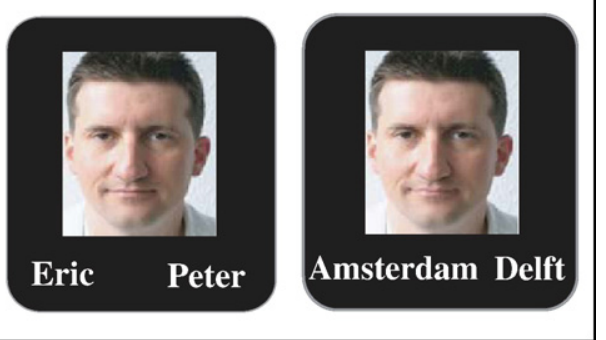

Fig. 1. Experimental design. During the study phase inside the scanner, participants were instructed to judge whether each first name and city name fitted well together with the face (presented as a color photograph during the experiment), and memorize each face-name-city triplet for the subsequent memory tests. Afterwards, outside the scanner, two memory tests were given. Firstly, participants performed a face-item recognition memory test for the 264 studied faces randomly intermixed with 132 unstudied faces using a 6-point confident rating scale (1-absolutely sure it was new/unstudied face; 6-absolutely sure it was studied/old face). Thereafter, studied faces that received a 6,5, or 4 rating (i.e., as the dotted ellipse shown) in the face-item recognition memory test were submitted to subsequent face-name and face-city associative recognition memory tests providing three response options: the left-side name (i.e., first name/ city) was associated with the face during study, unsure which name was associated with the face, the right-side name was associated with the face during study. In the associative recognition memory test, each face was shown with two previously studied names (gender selective) or two studied city names. One of the two names was previously presented together with the face; the other was presented with a different face at study and served as a foil (see Task procedure for more details). 
and simultaneous associations allows us to reveal the neural correlates of associative memory formation across time, while memory formation of single items and associations in general is equated.

\section{Materials and methods}

\section{Participants}

Twenty-six Dutch native, right-handed healthy university students (mean age $=22 \pm 4$ years; 8 males; $1-5$ years of university level education) without any neurological or psychiatric history and normal or corrected-to-normal vision participated in the experiment. All participants gave informed written consent according to the local ethics committee (CMO region ArnhemNijmegen, the Netherlands) and the declaration of Helsinki. We excluded data of six subjects, due to poor memory performance on the subsequent memory tasks (the correct rate of remembered simultaneous associations or discontinuous associations $<0.55$ ).

\section{Stimuli}

We selected 396 portraits (half males) from a database of 1000 color photographs of European individuals unknown to the participants (http:/www.ftd.de/static/1000europeans). To standardize the stimuli and minimize some potential confounding factors, faces were selected under several criteria such as direct gaze contact, no strong emotional facial expression, no headdress, no glasses, no beard, and so on. Familiar Dutch first names ( $n=264$; half males) and Dutch city names $(n=264)$ were obtained from the Internet. Length of names and cities ranged from 3 to 10 letters (mean length $(\mathrm{SD})=5.6$ (1.5) and 5.8 (1.7), respectively). We created 264 face-name-city triplets for the study phase and six triplets for a pre-scan training session. One hundred thirty-two faces were used as lures for the face item recognition memory test. Triplets were created randomly out of the entire set of 264 faces for each subject individually with gender congruent face-name pairings. Additionally, another six trials were created in order to familiarize the participants with the task prior to scanning.

\section{Task procedure}

The experiment consisted of a study phase and two subsequent memory tests. At study, participants were scanned while they were memorizing 264 face-name-city triplets, with the face common to two associates (i.e., a first name and a city). Each trial started with one of two associates (either a first name or a city) called 'Event 1', which was presented at the center of the screen for $2 \mathrm{~s}$. The first event was followed by a short delay period (3 s) during which a red question mark was presented. Thereafter, the second event ('Event 2') was composed of a face and the other associate (either a city or a first name), which was presented at the center of the screen for $4 \mathrm{~s}$, with the face presented above the associate (Fig. 1). To avoid possible bias effects of cognitive process and neural activity related to first names and cities, half of the trials started with a first name and the other half started with a city name, randomly intermixed. To make participants integrate all three items into one association, they were instructed to indicate by appropriate button-presses whether they thought that the face-name-city triplet fitted well together or not. In addition, they were explicitly instructed to memorize each face with the associated name and city name. Therefore, each trial consisted of a discontinuous association (the initial associate and the face separated by the delay period) and a simultaneous association (the associate presented together with the face). Trials were separated by an inter-trial-interval (ITI) of 3 to $6 \mathrm{~s}$ (varied in steps of $1 \mathrm{~s}$ ).

About $20 \mathrm{~min}$ after the study phase, subjects were asked to perform two memory tests outside the scanner. The first one was a face-item recognition memory test, in which the participants saw the 264 previously studied faces randomly intermixed with 132 new faces and were asked to judge whether the face was an old (i.e., studied) or a new (i.e., unstudied) face. Participants gave their old/new decision by a confidence rating on a 6-point scale $(1=$ absolutely sure it was a new face; $2=$ somewhat sure it was a new face; 3 =guessing it was a new face; $4=$ guessing it was an old face; $5=$ somewhat sure it was an old face; $6=$ absolutely sure it was an old face). This task was self-paced with a minimal trial duration of $2 \mathrm{~s}$ and a maximal one of $6 \mathrm{~s}$. Old faces that received a 6,5 , or 4 rating were included in the subsequent associative memory test. Here, each face was shown with two previously studied names (gender selective) or two studied city names. One of the two names was previously presented together with the face; the other was presented with a different face at study and served as a foil to ensure that the decision about the correct association was not based solely on familiarity. It should be noted that the number of associative recognition memory trials was always limited to 132 face-name and 132 face-city associations, because half of studied names (or cities) should be attributed to foils. Sixteen subjects had more than 132 hit trials with a ' 5 ' or ' 6 ' rating in the face item recognition memory test. In these subjects, we randomly selected out of these hit trials 132 faces for the associative memory test and the remaining faces were included in 'the condition of no interest'. Four subjects had less than 132 hit trials in the face item recognition memory test with a ' 5 ' or ' 6 ' rating. Hence, we randomly selected some studied faces with a ' 4 ' rating for their associative recognition memory test, but these trials were included in 'the condition of no interest'. Each face was presented once for each type of face-name and face-city association. To counterbalance the type and order, when presenting face-name and face-city associations in the associative recognition test, all 132 face-name and 132 face-city trials were randomly divided into two blocks, respectively, and counterbalanced across participants. The position of the correct name was balanced between the right and the left position on the screen. This task was self-paced, limited between 3 and $6 \mathrm{~s}$. Participants were asked to indicate by an appropriate button press, which name was associated with the presented face during the study phase. To exclude trials potentially based on guessing, participants were instructed to give an unsure response when they did not remember which association was the correct one.

\section{fMRI data acquisition}

During MRI scanning, whole head T2*-weighted EPI-BOLD fMRI data were acquired with a Siemens Sonata 1.5 T MR-scanner using an ascending slice acquisition sequence (33 axial slices, volume $\mathrm{TR}=2.29 \mathrm{~s}, \mathrm{TE}=30 \mathrm{~ms}, 90^{\circ}$ flip-angle, slice-matrix size $=64 \times 64$, slice thickness $=3.0 \mathrm{~mm}$, slice gap $=0.5 \mathrm{~mm}$, field of view $=224 \mathrm{~mm}$ ). Following the experimental session, highresolution structural images were acquired, using a T1-weighted MP-RAGE sequence (volume $\mathrm{TR}=2250 \mathrm{~ms}, \mathrm{TE}=3.93 \mathrm{~ms}, 15^{\circ}$ 
flip-angle, 176 sagittal slices, slice-matrix size $=256 \times 256$, slice thickness $=1 \mathrm{~mm}$, no slice gap, field of view $=256 \mathrm{~mm}$ ).

\section{fMRI data analysis}

Image pre-processing and statistical analysis was performed using SPM2 (http://www.fil.ion.ucl.ac.uk/spm). The first five volumes of each participant's EPI-data were discarded to allow for T1 equilibration. The subject's structural MR image was coregistered to the mean of the functional images, using mutual information optimization. Subsequently, the functional images were slice-time corrected, spatially normalized and transformed into a common stereotactic space, as defined by the SPM2 MNI T1 template, as well as spatially filtered by convolving the functional images with an isotropic 3D Gaussian kernel (10 mm FWHM). The fMRI data were statistically analyzed using the general linear model and statistical parametric mapping (Friston et al., 1995). In addition, the realignment parameters were included to account for movement-related variability. The data were high-pass filtered (128 s) to account for various low-frequency effects.

Tackling the question at issue, the encoding trials were sorted into several different categories based on the subsequent memory performance (Table 1). Our main interest was the transient neural activity when associative memory formation occurred; therefore, separate regressors of each category were created time-locked to the onset of 'Event 2', and convolved with the canonical hemodynamic response function. 'Event 1' was separately modeled independently of subsequent memory performance to account for encoding-related neural responses to the first event. According to the recognition memory performance (see Behavioral Results), a '1', '2', or ' 3 ' rating for an old face can be regarded as forgotten (i.e., 'Miss' condition) and a ' 5 ' or ' 6 ' rating as remembered. Trials associated with remembered items were subdivided in five different conditions, depending on the subsequent associative recognition memory performance: (1) Hit single item: correct face recognition, incorrect associative recall; (2) Hit simultaneous association: correct face recognition, only correct associative recall for the simultaneously memorized name or city name; (3) Hit discontinuous association: correct face recognition, only correct associative recall for the discontinuously memorized name or city name; (4) Hit all associations (i.e., hit both simultaneous and discontinuous associations): correct face recognition, correct associative recall for the simultaneously and discontinuously memorized name or city name; (5) Hit of no interest: correct face recognition, unsure on both associated name and associated city, miss on either associated name or associated city but unsure on either associated name or associated city, as well

Table 1

Number of trials and reaction times (mean \pm S.D.) of the five categories of main interest $(n=20)$

\begin{tabular}{lccccc}
\hline & Miss & Single & Simul. & Discon. & All \\
\hline Num. & $69.8(19.5)$ & $14.5(5.8)$ & $30.3(7.2)$ & $30.9(5.3)$ & $39.6(11.5)$ \\
RTs (ms) & $2107(465)$ & $2035(499)$ & $2038(423)$ & $2026(442)$ & $2001(396)$ \\
\hline
\end{tabular}

'Miss' - forgotten faces, 'Single' - hit single item: correct face recognition and incorrect associative recall, 'Simul.'- - hit simultaneous association, 'Discon.' - hit discontinuous association, 'All' — hit both simultaneous and discontinuous associations, 'Num.' - number of trials. The number is out of 264 in the condition of 'Miss'. In contrast, the numbers are out of 132 in the remaining conditions (see fMRI data analysis for details). as the aforementioned trials in 'the condition of no interest' (see Task procedure). Additionally, a low level fixation condition was separately modeled.

The relevant contrast parameter images generated on the single participant level were submitted to the second-level group analysis. At the group level, contrasts between conditions were computed by using one-sample $t$ tests on these images treating participants as a random variable. In the whole brain search, the results from the random effects analyses were initially threshold at $p<0.001$ (uncorrected) and the cluster size statistics were used subsequently as the test statistic. Only clusters significant at $p<0.05$ (corrected for multiple non-independent comparisons; Worsley et al., 1996) are reported. All local maxima (MNI coordinates) within significant clusters are reported with $p$ values corrected for multiple non-independent comparisons based on false discovery rate (Genovese et al., 2002), unless otherwise specified. Given that the MTL is an obvious region of interest, the MTL was additionally investigated within a spherical region of interest (radius $=25 \mathrm{~mm}$ ) centered in the middle of the hippocampus at $[x, y, z]=[ \pm 30,-22,-14]$ in combination with a small volume correction (SVC) for multiple non-independent comparisons.

To characterize the patterns of activation related to the subsequent memory effects for different types of memory formation within the MTL, we further conducted regions of interest (ROIs) analysis in the anterior and posterior MTL (Schacter and Wagner, 1999; Sperling et al., 2003; Jackson and Schacter, 2004). Using MarsBar (http://www.marsbar.sourceforge.net; Brett et al., 2002), we functionally identified the ROIs based on the average group activation within the MTL. Each ROI included all voxels within a sphere radius of $6 \mathrm{~mm}$ centered at a certain local maxima located in the MTL (see Results). Averaged effect sizes of all voxels within the ROIs were extracted by estimating the mean contrast values of all individual subjects.

\section{Results}

\section{Behavioral results}

\section{Study phase}

Participants were more likely to judge the face-name-city association as a good fit than not $(0.696 \pm 0.143$ vs. $0.304 \pm 0.143$; $\left.t_{(19)}=6.01, p<0.001\right)$. They performed equally fast in that half of the encoding trials that started with a city name (2062 $\pm 448 \mathrm{~ms})$ and in the other half of trials that started with a first name $(2041 \pm 417 \mathrm{~ms})\left(t_{(19)}=0.95 ; p>0.10\right)$. This suggests that both trial types were similar to each other in difficulty.

\section{Face item recognition memory test}

The distribution of averaged response proportions (mean $\pm \mathrm{SE}$ ) is shown in Fig. 2. Two separate repeated-measures ANOVAs revealed significant differences of confidence ratings for both old and new faces $\left(F_{(5,95)}=19.24, F_{(5,95)}=7.10\right.$, respectively; both $p$ values $<0.001)$. Paired-sample $t$ tests comparing confidence ratings revealed that the proportion of ' 5 ' and ' 6 ' ratings for old faces were significantly higher than for new faces $\left(t_{(19)}=6.97, t_{(19)}=9.07\right.$, respectively; both $p$ values $<0.001$ ). The proportion of ' 4 ' ratings was similar for old and new faces $\left(t_{(19)}=0.66 ; p=0.54\right)$. Reversely, the proportion of ' 1 ', ' 2 ', and ' 3 ' ratings for old faces was lower than for new faces $\left(t_{(19)}=-5.53, t_{(19)}=-6.00, t_{(19)}=-4.79\right.$ respectively; all $p$ values $<0.001)$. The performance was independent of 'fit' and 


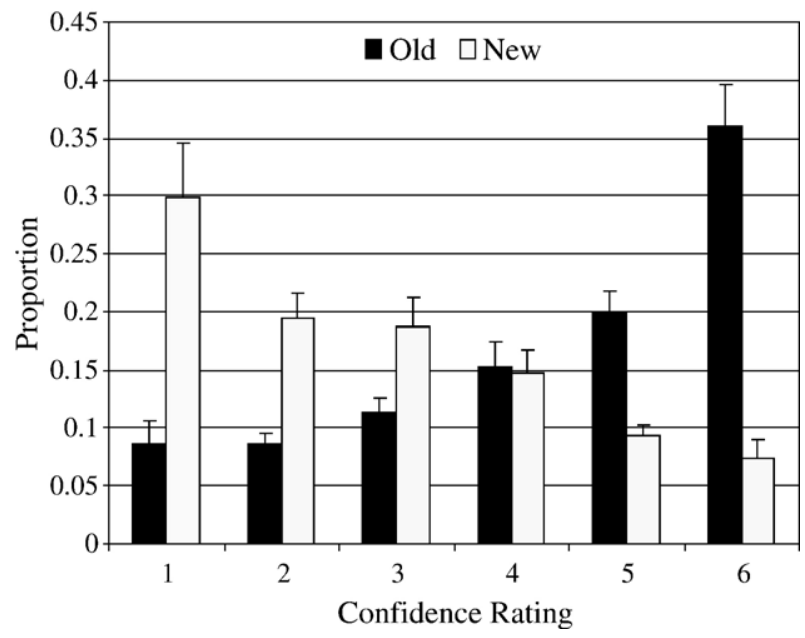

Fig. 2. Subsequent face-item recognition memory performance $(n=20)$. Distributions of mean hit and false alarm rates: Mean (S.E.) proportions of responses are shown on the $y$-axis and confident ratings are depicted on the $x$-axis.

'not fit' responses. ${ }^{1}$ These results demonstrate successful discrimination between studied and new faces.

\section{Associative memory test}

Number of trials and reaction times of the five categories of main interest are shown in Table 2. The rate $($ mean $\pm S D)$ of correctly recalled associations was $0.626 \pm 0.063$ for simultaneous associations and $0.629 \pm 0.069$ for discontinuous associations. This level of associative memory performance was roughly consistent with previous studies using similar subsequent memory paradigm (Sperling et al., 2003; Chua et al., 2004). More importantly, the performance on both types of associations was significantly above chance level $\left(t_{(19)}=8.98 ; t_{(19)}=8.34\right.$, respectively; both $p$ values $<0.001$ ) and did not differ from one another $\left(t_{(19)}=-0.09 ; p=0.93\right)^{2}$. Given that the associated name and associated city name were simultaneously or discontinuously presented with the face, we constructed two (presenting types: simultaneous and discontinuous)-by-two (pairing types: facename and face-city) repeated-measure ANOVAs to confirm that there was no interaction between these two factors for the performance $\left(F_{(1,19)}<1.0\right)$.

Finally, we confirmed that the response in the orientation task had no significant effect on associative memory performance (i.e., 'Hit simultaneous association': fit vs. not fit $=0.128$ vs. 0.117 ; 'Hit discontinuous association': fit vs. not fit $=0.128$ vs. 0.118 ; 'Hit all associations': fit vs. not fit $=0.162$ vs. 0.144 ; all $p$ values $>0.13$ ). Thus, we collapsed for all further analysis fit and not fit trials to increase statistical power.

\footnotetext{
1 The response in the orientation task did not predict face item recognition memory performance (i.e. 'Miss' faces-1, 2 and 3 ratings: fit vs. not fit $=0.228$ vs. 0.229 ; 'Remembered' faces -5 and 6 ratings: fit vs. not fit $=0.475$ vs. 0.438 ; all $p$ values $>0.30$ ).

2 To reduce contamination by guesses as much as possible, we excluded trials that received an unsure response in the subsequent memory tests and excluded those subjects with a mean associative memory performance of 0.55 or less (see above).
}

\section{Neuroimaging results}

Neural correlates of the formation of discontinuous associations

To identify brain regions specifically related to the formation of discontinuous associations, we directly contrasted the conditions 'hit discontinuous association' with 'hit simultaneous association'. Thus, the contrast of two conditions does not differ in the number of associations formed but the type of association formed. A separate set of brain regions was specifically more active for the formation of new discontinuous associations (Table 2; Figs. 3A and B). In more detail, these regions included the left posterior parahippocampal gyrus close to the junction between the fusiform gyrus and the parahippocampal gyrus (local maximum at $[-33$, $-39,-9$ ], $Z=4.42$; cluster $p=0.007 \mathrm{SVC}$ corrected); a large area in the dorsolateral prefrontal cortex including the right superior frontal gyrus (local maximum at [15, 39, 33], $Z=4.28$; [33, 30, 39], $Z=4.04$; $[15,48,30], Z=3.94$; all cluster $p$ values $<0.05$ corrected) and the right middle frontal gyrus (local maximum at [33, 30, 39], $Z=3.74$; cluster $p<0.05$ corrected); the basal ganglia including the caudate nucleus and the putamen (local maxima at $[-18,9,12]$, $Z=4.72 ;[21,6,6], Z=3.98 ;[27,9,15], Z=3.96 ;[-21,12,-3]$, $Z=3.87 ; \quad[-18,3,-6], Z=3.18$; all cluster - values $<0.05$ corrected); a medial posterior region including the precuneus and the posterior cingulate gyrus (local maximum at $[15,-60,18]$, $Z=3.94$; cluster $p<0.05$ corrected); and the occipital-temporal region from the posterior middle temporal gyrus extending into the superior occipital gyrus (local maxima at $[-39,-63,15], Z=3.85$; $[-42,-69,27], Z=3.65 ;[-33,-81,36], Z=3.47$; all cluster $p$ values $<0.05$ corrected). There were no significant activations observed for the opposite contrast. The ROI analysis revealed a strong linear increase of the subsequent memory effect in the posterior parahippocampal gyrus from 'only hit single item', 'only hit simultaneous association' to 'only hit discontinuous association' $\left(F_{(2,38)}=5.53, p=0.008\right)$. Hence, we identified a set of brain regions that is specifically involved in transferring associations of discontinuous events into episodic memory. This network includes the posterior parahippocampal region, the dorsal-lateral prefrontal cortex, posterior midline structures, and the basal ganglia.

\section{Neural correlates of the formation of new memories including associations}

To replicate the neural correlates of associative memory formation, we contrasted brain activity associated with successful associative memory formation (i.e., hit simultaneous association, hit discontinuous association, and hit all associations) and nonsuccessful memory formation (i.e., misses). We identified a wellestablished set of regions that were significantly more active during the successful formation of new associations, including the prefrontal cortex and the occipito-temporal cortex extending into the MTL (Table 3; Fig. 4A). In the MTL, we identified the left posterior hippocampus (local maximum at $[-24,-33,-3], Z=4.75$; $[-18,-36,3], Z=4.10 ; p=0.012$ corrected), the left anterior hippocampus extending into the amygdala and the parahippocampal gyrus (local maximum at $[-21,-6,-18], Z=3.84 ; p=0.014$ corrected), and the right anterior to middle hippocampus extending into the amygdala (local maxima at $[24,-6,-18], Z=4.35$ and [21, $-27,-9], Z=3.58$; all $p<0.015$ corrected). In the prefrontal cortex, we identified the ventral medial frontal gyrus (local maximum at $[0$, $45,-18], Z=4.08 ; p=0.012$ corrected), the bilateral superior frontal gyrus (local maxima at $[3,60,24], Z=3.67 ;[-9,54,24], Z=3.54$; all $p=p=0.017$ corrected), and the left and right inferior frontal 
Table 2

Brain regions associated with the formation of discontinuous associations: Hit discontinuous associations vs. hit simultaneous associations $(n=20)$

\begin{tabular}{|c|c|c|c|c|c|c|}
\hline \multirow[t]{2}{*}{ Brain region } & \multirow[t]{2}{*}{$\mathrm{BA}$} & \multirow{2}{*}{$\frac{\text { Cluster }}{p \text { corrected }}$} & \multirow[t]{2}{*}{$Z$ value } & \multicolumn{3}{|l|}{ MNI } \\
\hline & & & & $x$ & $y$ & $z$ \\
\hline \multirow[t]{3}{*}{ R. superior, medial/middle frontal gyri } & \multirow[t]{3}{*}{8,9} & \multirow[t]{3}{*}{$<0.0001$} & 4.28 & 15 & 39 & 33 \\
\hline & & & 4.04 & 33 & 30 & 39 \\
\hline & & & 3.94 & 15 & 48 & 30 \\
\hline L. posterior parahippocampal gyrus & 35 & $0.007 *$ & 4.42 & -33 & -39 & -9 \\
\hline \multirow[t]{3}{*}{ L. caudate, lentiform nucleus, putamen } & \multirow[t]{3}{*}{-} & \multirow[t]{3}{*}{0.004} & 4.72 & -18 & 9 & 12 \\
\hline & & & 3.87 & -21 & 12 & -3 \\
\hline & & & 3.18 & -18 & 3 & -6 \\
\hline \multirow[t]{2}{*}{ R. caudate, lentiform, putamen } & \multirow[t]{2}{*}{-} & \multirow[t]{2}{*}{0.043} & 3.98 & 21 & 6 & 6 \\
\hline & & & 3.96 & 27 & 9 & 15 \\
\hline R. precuneus, posterior cingulate cortex & 31 & 0.004 & 4.05 & 15 & -60 & 18 \\
\hline \multirow[t]{2}{*}{ L. middle temporal gyrus } & 19,39 & \multirow[t]{3}{*}{0.043} & 3.85 & -39 & -63 & 15 \\
\hline & 39 & & 3.65 & -42 & -69 & 27 \\
\hline L. superior occipital gyrus & 19 & & 3.47 & -33 & -81 & 36 \\
\hline
\end{tabular}

The threshold $p<0.001$ (uncorrected) in the whole brain volume search. Only clusters ( 20 or more extent voxels) significant at $p<0.05$ (corrected cluster) were reported. *Small Volume Correction (SVC) used in the MTL activations. L: left; R: right; BA: Brodmann Area; MNI: MNI coordinates (SPM2). The activations in MTL are in bold type.

gyrus (local maxima at $[-42,33,-9], Z=3.53 ;[42,30,12], Z=3.64$; $Z<0.024)$. To further characterize the patterns of activation related to the subsequent memory effects for different types of memory formation, we analyzed the bilateral anterior and posterior hippocampus using ROI analyses. As shown in Fig. 4B, there is a trend toward a linear increase of subsequent memory in the bilateral anterior hippocampus from 'hit single item', 'hit simultaneous association', 'hit discontinuous association' to 'hit all associations' (Left: $F_{(3,57)}=2.35, p=0.082$; Right: $F_{(3,57)}=2.85, p=0.045$ ). Hence, these findings confirm previous neuroimaging studies (Dolan and Fletcher, 1997; Henke et al., 1997; Sperling et al., 2003; Jackson and Schacter, 2004; Mitchell et al., 2004; Achim and Lepage, 2005; Prince et al., 2005) showing that specific prefrontal and medial temporal structures including the hippocampus are involved in associative or relational memory formation. It is worthwhile to note that there was an inadequate number of trials in the 'hit single item' condition (i.e., mean: $14.5 \pm 5.8$ ) for powerful statistical analyses, therefore this condition would introduce an unacceptable level of noise if being compared to other associative memory conditions.

To examine whether the hippocampus was actually activated in each of the three conditions of associative memory formation (i.e., 'hit simultaneous association', 'hit discontinuous association', and 'hit all associations'), we constructed three separate contrasts against the 'misses'. For the formation of simultaneous associations, we found the left posterior hippocampus (local maxima at

Table 3

Brain regions related to associative memory formation: formation of new memories including associations vs. Miss

\begin{tabular}{|c|c|c|c|c|c|c|c|}
\hline \multirow[t]{2}{*}{ Brain region } & \multirow[t]{2}{*}{$\mathrm{BA}$} & \multirow{2}{*}{$\frac{\text { Cluster }}{p \text { (corrected) }}$} & \multirow{2}{*}{$\frac{\text { Voxel }}{P_{\text {FDR }}}$} & \multirow[t]{2}{*}{$Z$ score } & \multicolumn{3}{|l|}{ MNI } \\
\hline & & & & & $x$ & $y$ & $z$ \\
\hline \multirow[t]{2}{*}{ R. middle occipital, lingual and fusiform gyri } & \multirow[t]{2}{*}{$18 / 19$} & \multirow{2}{*}{$<0.0001$} & 0.012 & 4.83 & 36 & -72 & -9 \\
\hline & & & 0.012 & 4.58 & 30 & -90 & 15 \\
\hline R. inferior occipital gyrus & 18 & & 0.012 & 4.45 & 30 & -87 & -9 \\
\hline L. lingual gyrus & 18 & & 0.012 & 4.22 & -9 & -99 & 3 \\
\hline L. inferior occipital gyrus & $17 / 18$ & & 0.012 & 4.05 & -30 & -93 & -6 \\
\hline \multirow[t]{2}{*}{ L. hippocampus, parahippocampal gyrus } & \multirow[t]{2}{*}{$27 / 30$} & \multirow{2}{*}{0.010} & 0.012 & 4.75 & -24 & -33 & -3 \\
\hline & & & 0.012 & 4.10 & -18 & -36 & 3 \\
\hline \multirow[t]{2}{*}{ R. hippocampus, parahippocampal gyrus } & \multirow[t]{2}{*}{35} & \multirow[t]{2}{*}{$<0.0001$} & 0.012 & 4.35 & 24 & -6 & -18 \\
\hline & & & 0.015 & 3.76 & 21 & -27 & -9 \\
\hline \multirow[t]{3}{*}{ Ventral medial frontal gyrus } & \multirow[t]{3}{*}{$11 / 10$} & \multirow[t]{3}{*}{0.008} & 0.012 & 4.08 & 0 & 45 & -18 \\
\hline & & & 0.028 & 3.30 & -6 & 60 & -12 \\
\hline & & & 0.038 & 3.12 & 15 & 36 & -15 \\
\hline L. fusiform and parahippocampal gyri & 37 & 0.012 & 0.013 & 3.96 & -36 & -51 & -15 \\
\hline \multirow[t]{2}{*}{ L. anterior hippocampus, amygdala } & \multirow[t]{2}{*}{-} & \multirow[t]{2}{*}{0.007} & 0.014 & 3.84 & -21 & -6 & -18 \\
\hline & & & 0.015 & 3.79 & 0 & 6 & -9 \\
\hline R. superior frontal gyrus & \multirow[t]{2}{*}{9,10} & \multirow[t]{2}{*}{0.051} & 0.017 & 3.67 & 3 & 60 & 24 \\
\hline L. superior frontal gyrus & & & 0.017 & 3.54 & -9 & 54 & 24 \\
\hline R. inferior frontal gyrus & 46 & 0.351 & 0.024 & 3.64 & 42 & 30 & 12 \\
\hline L. inferior frontal gyrus & 47 & 0.882 & 0.020 & 3.53 & -42 & 33 & -9 \\
\hline R. inferior/middle frontal gyrus & 47 & 0.786 & 0.020 & 3.53 & 36 & 33 & -15 \\
\hline
\end{tabular}

The threshold $p<0.05$ (corrected) in the whole brain search. Only clusters (10 or more extent voxels) significant at $p<0.05$ (corrected) were reported. L: left; R: right; BA: Brodmann Area; MIN: MIN coordinates (SPM2). The activations in MTL are in bold type. 


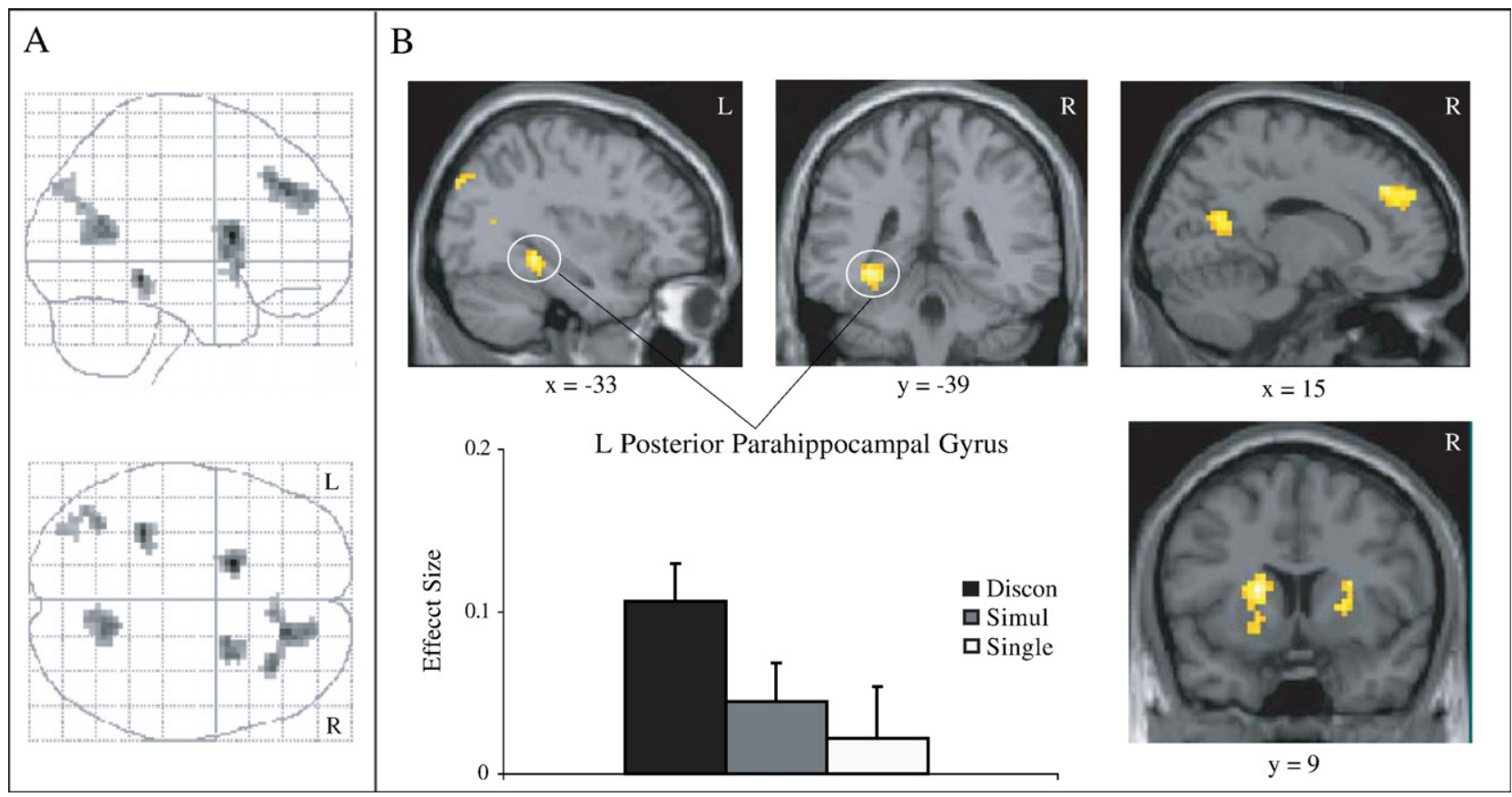

Fig. 3. Brain regions showing activation related specifically to the formation of new discontinuous associations. (A) Intensity projection of whole brain activity related to the contrast between 'hit discontinuous associations' vs. 'hit simultaneous associations'. (B) Selected activation clusters superimposed on corresponding T1 images in the left posterior parahippocampal gyrus (left and middle upper panel), the dorsolateral prefrontal cortex and precuneus/posterior midline region (right upper panel), basal ganglia including the bilateral caudate/putamen (right bottom panel). The bar graph shows the averaged effect size (S.E.) of subsequent memory effects in the posterior parahippocampal gyrus centered at $[-33,-39,-9]$. For displaying purposes, maps are thresholded at $p<0.001$ (uncorrected, $\geq 20$ voxels). R, right; L, left.

$[-24,-33,-3],[-27,-30,-6]$, all $Z>3.46$; cluster $p=0.024 \mathrm{SVC}$ corrected), the left anterior hippocampus (local maxima at $[-30,0$, $-15],[-24,-9,-21]$, all $Z>3.28$; cluster $p=0.054$ SVC corrected), and the right anterior hippocampus/amygdala (local maxima at $[24,-6,-18], Z=4.11$; cluster $p=0.008 \mathrm{SVC}$ corrected) to be significantly activated. For the formation of discontinuous associations, we found the left posterior hippocampus extending into parahippocampal and fusiform gyri (local maxima at $[-24$, $-36,-6],[-27,-33,-9]$, and $[-30,-45,-15]$, all $Z>3.93$; cluster $p<0.001$ SVC corrected), the left anterior hippocampus (local maxima at $[-21,-6,-12], Z=3.58$; cluster $p=0.019 \mathrm{SVC}$ corrected), the right anterior hippocampus/amygdala (local maxima at $[21,-3,-21],[33,0,-15]$ and $[24,-12,--15]$, all $Z>3.49$; cluster $p=0.001$ corrected), and the right posterior hippocampus (local maxima at $[21,-27,-9], Z=3.77$; cluster $p=0.037 \mathrm{SVC}$ corrected) to be significantly activated. For the formation of simultaneous and discontinuous association, we found the left posterior hippocampus (local maxima at $[-27,-30,-9]$ and $[-24$, $-33,-3], Z>3.66$; cluster $p=0.019$ SVC corrected) and bilaterally the anterior hippocampus/amygdala (local maxima at $[-21,-6$, -18], $Z=3.37$; cluster $p=0.060$ SVC corrected; [24, -9, -12], $Z=3.90$; cluster $p=0.009$ SVC corrected) to be significantly activated. Hence, the hippocampal contribution appears independent of the type of association formed. Additionally, to identify the neural activity specifically related to remembering all associations, we conducted the contrast of 'hit all associations' vs. 'hit simultaneous association' and 'hit discontinuous association'. When testing the whole brain data $(p<0.001$ uncorrected), we found an almost significant effect in the ventral medial prefrontal cortex only (local maxima at $[3,51,-15], Z=3.90 ;[-3,24,-18]$,
$Z=3.83 ; \quad[-12,24,-12], Z=3.83 ;$ cluster $p$ values $<0.08$ corrected). However, there was not a reliable effect in the MTL; this result indicates that the activity of MTL was not significantly greater in remembering all associations than remembering either simultaneous association or discontinuous association.

\section{Discussion}

The goal of this study was to reveal the neural correlates of a specific type of associative memory formation, the binding of events that are separated in time. We show that activity in the left posterior parahippocampal gyrus, the dorsolateral prefrontal cortex, posterior midline structures, and certain parts of the basal ganglia is increased while subjects successfully store discontinuous associations into episodic memory. This finding suggests that there is a distinct (set of) operation(s) that supports specifically an essential function for episodic memory, the integration of inter-item associations across time. Importantly, this operation might be functionally dissociated from operations that form associative memories in general.

To bind two discontinuous events together, it is necessary to keep the initial event online until the second event is perceived and/or to retrieve/reactivate the representation of the initial event while processing the second event. Thus, one probability is that the online maintenance operation might be responsible for our results in part. However, our findings cannot readily be attributed to the online maintenance of the initial event only, a word, because we analyzed the data event-related to the second event and the neural responses of the initial event were accounted for in the design model. Furthermore, active maintenance of words is 


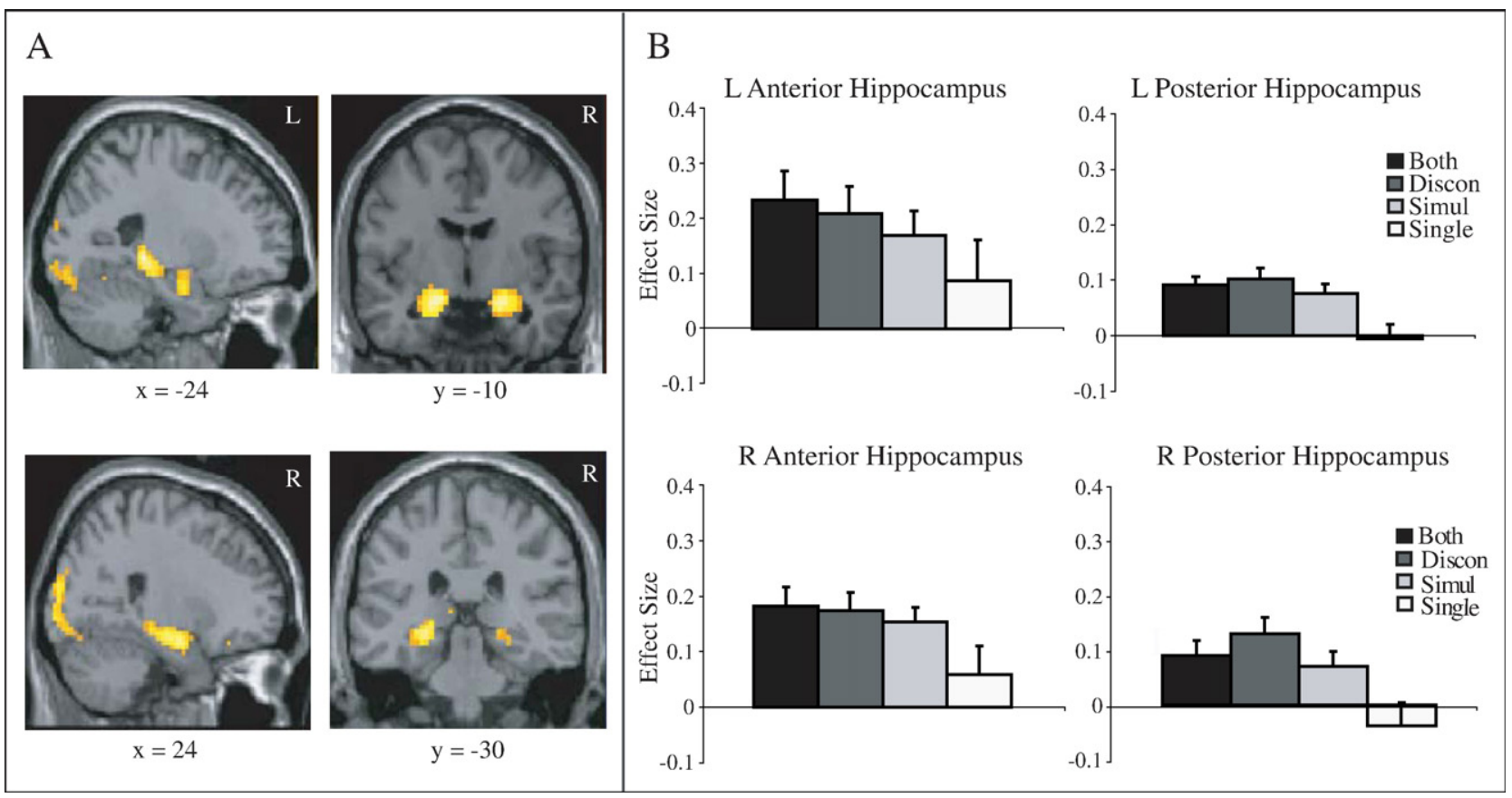

Fig. 4. Brain regions showing significant activation for successful formation of new memories including associations. (A) Regions in the MTL activated by the contrast between 'Formation of new memories including associations' and 'Misses': the left anterior and posterior hippocampus (left upper panel—sagittal view), the right anterior-to-posterior hippocampus (left bottom panel—sagittal view), the bilateral anterior hippocampus extending into amygdala (right upper panel — coronal view), and the bilateral posterior hippocampus (right bottom panel—coronal view) (all $p<0.05$ FDR corrected). (B) It shows the averaged effect size (S.E.) of subsequent memory effects for different types of memory formation, regions of interest derived from the group activation of 'Formation of new memories including associations' and 'Misses': the bilateral anterior hippocampus centered at $[ \pm 24,-6,-18]$ (left panels), the bilateral posterior hippocampus centered at $[-24,-33,-3]$ and $[21,-27,-9]$, respectively (right panels). For displaying purposes, maps are thresholded at $p<0.05$ (FDR corrected, $\geq 20$ voxels). R, right; L, left.

typically associated with activity in a left hemispheric set of brain regions including the inferior prefrontal cortex and the temporal-parietal junction (Prabhakaran et al., 2000; Sakai et al., 2002a; Sakai and Passingham, 2004; Narayanan et al., 2005; Hart et al., 2006). Thus, our results can be more readily attributed to an actual binding operation, a retrieval/reactivation operation or both.

The posterior parahippocampal area has often been implicated in actual binding (Davachi et al., 2003; Ranganath et al., 2004a,b; Prince et al., 2005; Sommer et al., 2005a, 2005b), processing contextual associations (Aminoff et al., 2006), and long-term memory formation (Brewer et al., 1998; Wagner et al., 1998; Schon et al., 2004, 2005). Consistently, Eichenbaum (2000) concluded from animal experiments that parahippocampal regions are involved in the encoding of associations into an integrated representation. In non-human primates, the inferior temporal cortex (consisting of two distinct but mutually interconnected areas: area TE and area 36), including the parahippocampal region, has been proposed to be involved in associative long-term memory formation of visual information (Squire and Zola-Morgan, 1991; Murray et al., 1993; Miyashita, 2000; Messinger et al., 2001). However, this does not contradict the well-established view that the hippocampus is critically involved in the formation of associative memories. Our results also confirm that the hippocampus is generally activated when new associative memories including simultaneous association and discontinuous associations are successfully formed. The posterior parahippocampal area seems to play a specific role when discontinuous associations are successfully encoded into episodic memory.

The dorsolateral prefrontal cortex (together with the basal ganglia) has been associated with the maintenance and associative binding of complex information, including across-temporal associations (Fuster et al., 2000; Prabhakaran et al., 2000; Pasupathy and Miller, 2005), and the top-down control of memory retrieval/reactivation (Miyashita, 2004). For instance, lesions including the dorsolateral prefrontal cortex in humans and nonhuman primates impair performance of specific working memory tasks like the associative learning task (i.e., forming and rearranging of arbitrary associations) (Petrides, 1985, 1997; Asaad et al., 1998). Numerous functional neuroimaging studies have confirmed that the dorsolateral prefrontal cortex contributes to executive control processes such as selection and active maintenance in working memory (Rowe et al., 2000; Sakai et al., 2002b; Curtis and D'Esposito, 2003, 2006; Curtis et al., 2004). Moreover, some recent studies found that this brain region contributes to long-term memory formation through its role in organizing information in working memory (Wagner, 1999; Blumenfeld and Ranganath, 2006). Several lines of evidence also have suggested that the dorsolateral prefrontal cortex interacts closely with the basal ganglia when complex information has to be maintained in working memory (Levy et al., 1997; Ashby et al., 2005; Pasupathy and Miller, 2005; Chudasama and Robbins, 2006; O'Reilly and Frank, 2006). Hence, our finding of dorsolateral prefrontal and caudate nucleus activity related to the successful 
encoding of discontinuous events might be explained by higher organizational demands during associative binding.

An alternative, yet not mutually exclusive explanation for the dorsolateral prefrontal contribution might be related to the top-down control of memory retrieval/reactivation. Previous lesion studies, single-unit neurophysiological, and functional neuroimaging studies have suggested that the dorsolateral prefrontal cortex is the source of top-down signals that reactivate neural representations in posterior cortical areas like parietal and occipital midline structures (Miyashita and Hayashi, 2000; Ranganath and D'Esposito, 2005). In addition, the medial temporal lobe also appears to be involved in top-down control of memory retrieval/reactivation, but by another aspect (Miyashita and Hayashi, 2000; Ranganath and D'Esposito, 2005). For instance, the medial temporal activity spreading backwards reactivates relevant neurons in more posterior inferior temporal regions (Naya et al., 1996, 2001). While signals from the parahippocampal cortex during retrieval may actually trigger the reactivation of relevant information whenever rehearsal and thus active working-memory maintenance were interrupted, signals from the prefrontal cortex are always engaged in the selection of taskrelevant information in order to guide behavior (Sakai et al., 2002a; Sakai, 2003; Sakai and Passingham, 2004). Taken together, the dorsolateral prefrontal and posterior parahippocampal activation specifically related to the successful encoding of discontinuous events might reflect that these two areas trigger the reactivation of the representation of the initial event, allowing it to be successfully associated with the second event, in order to form a new association across time into episodic memory.

As shown by our data, posterior midline structures like the precuneus/posterior cingulate cortex are also involved in the formation of discontinuous associations. In the formation of discontinuous associations, when receiving top-down signals this brain region might mediate reactivation/retrieval of the internal representation of the initial event and attention orientations (i.e., directing attention to the internal representation of the initial event, shifting attention between the initial event and the second event) (Cavanna and Trimble, 2006). For example, activity in posterior midline structures is often reported by functional neuroimaging studies to be related to memory retrieval (Fletcher et al., 1995a,b, 1996; Cabeza et al., 1997; Cabeza and Nyberg, 2000; Wheeler et al., 2000, 2006; Wheeler and Buckner, 2003; Wagner et al., 2005) and attention orientation tasks (Nagahama et al., 1999). The activity of these regions could play a role in perceptual reactivation process during remembering and memory retrieval as necessary for episodic memories (Wheeler et al., 2000, 2006). The occipital-temporal region including the middle temporal gyrus and superior occipital gyrus could be related to visual processing of individual aspects of a discontinuous association during associative operations (Yonelinas et al., 2001; Mather et al., 2006). Moreover, one recent neuroimaging study suggests that the posterior brain regions like intra-parietal sulcus, precuneus, posterior cingulate cortex and superior temporal gyrus are also involved in the multifeatural contextual encoding of disparate features of an episode into a common memory representation (Uncapher et al., 2006).

However, several theories suggest that the hippocampus is critically engaged in bridging discontinuities across time (Rawlins, 1985; Wallenstein et al., 1998; Eichenbaum, 2004). The hippocampal coding of local context is thought to be important for accurate sequence encoding (Wallenstein et al., 1998; Eichenbaum, 2004; Kumaran and Maguire, 2006). Similarly, a recent event- related fMRI study confirms that the hippocampus is involved in the process of 'discontinuity association', bridging a delay between the constituents of word-pairs in anticipation of a semantic relatedness judgment (Luo and Niki, 2005). However, this finding was not directly related to memory formation. Although our present results replicate that the hippocampus plays a general role in storing new associations (Sperling et al., 2003; Jackson and Schacter, 2004; Prince et al., 2005; as well as reviews, Eichenbaum, 2000, 2004), we did not observe a preference for the formation of discontinuous associations. However, our task differs significantly from typical sequence encoding tasks, in which the order of items and thus contextual coding is most critical. This contextual coding is thought to be mediated by the hippocampus that creates 'context units', which do not represent particular items, but instead support the 'glueing' together of sequential items (Wallenstein et al., 1998; Eichenbaum, 2004; Kumaran and Maguire, 2006).

In conclusion, our main finding is that there is a (set of) operation(s) that specifically stores discontinuous events into memory, a fundamental supplement to previous studies on episodic memory formation. We suggest that, under goal-directed long-term memory formation, top-down signals from the prefrontal cortex and the MTL may trigger reactivation of internal representations in posterior midline structures of the first event (Tomita et al., 1999; Miyashita and Hayashi, 2000; Naya et al., 2001; Sakai et al., 2002a; Sakai, 2003; Sakai and Passingham, 2004; Miyashita, 2004), thus allowing it to be associated with the second event. At the same time, the dorsolateral prefrontal cortex together with the basal ganglia may support this encoding operation by executive and binding processes within working memory (Prabhakaran et al., 2000; Pasupathy and Miller, 2005). Finally, the posterior parahippocampal area may play a role in binding and actual forming new memory traces. Future studies, however, have to dissociate the functional roles of these brain regions implicated here in the transformation of discontinuous association into episodic memory.

\section{Acknowledgments}

This work was supported by the Royal Netherlands Academy of Arts and Sciences and the Chinese Academy of Sciences. We thank Paul Gaalman, Dr. Atsuko Takashima, and Maja Stulemeijer for their technical assistance in acquiring the MRI data, and Mr. Jianhui $\mathrm{Wu}$ for helpful discussions. We also thank all subjects for their participation in this study.

\section{References}

Achim, A.M., Lepage, M., 2005. Neural correlates of memory for items and for associations: an event-related functional magnetic resonance imaging study. J. Cog. Neurosci. 17, 652-667.

Aminoff, E., Cronau, N., Bar, M., 2006. The parahippocampal cortex mediates spatial and nonspatial associations. Cereb. Cortex 21 (Advance Access published).

Asaad, W.F., Rainer, G., Miller, E.K., 1998. Neural activity in the primate prefrontal cortex during associative learning. Neuron 21, 1399-1407.

Ashby, F.G., Ell, S.W., Valentin, W., Casale, M.B., 2005. FROST: a distributed neurocomputational model of working memory maintenance. J. Cogn. Neurosci. 17, 1728-1743.

Blumenfeld, R.S., Ranganath, C., 2006. Dorsolateral prefrontal cortex promotes long-term memory formation through its role in working memory organization. J. Neurosci. 26, 916-925. 
Brett, M., Johnsrude, I.S., Owen, A.M., 2002. The problem of functional localization in the human brain. Nat. Rev. Neurosci. 3, 243-249.

Brewer, J.B., Zhao, Z., Desmond, J.E., Glover, G.H., Gabrieli, J.D., 1998. Making memories: brain activity that predicts how well visual experience will be remembered. Science 281, 1185-1187.

Buckner, R.L., Kelley, W.M., Petersen, S.E., 1999. Frontal cortex contributes to human memory formation. Nat. Neurosci. 2, 311-314.

Cabeza, R., Nyberg, L., 2000. Neural bases of learning and memory: functional neuroimaging evidence. Curr. Opin. Neurol. 13, 415-421.

Cabeza, R., Mangels, J., Nyberg, L., Habib, R., Houle, S., McIntosh, A.R., Tulving, E., 1997. Brain regions differentially involved in remembering what and when: a PET study. Neuron 19, 863-870.

Cavanna, A.E., Trimble, M.R., 2006. The precuneus: a review of its functional anatomy and behavioral correlates. Brain 129, 564-583.

Chua, E.F., Rand-Giovannetti, E., Schacter, D.L., Albert, M.S., Sperling, R.A., 2004. Dissociating confidence and accuracy: functional magnetic resonance imaging shows origins of the subjective memory experience. J. Cogn. Neurosci. 16, 1131-1142.

Chudasama, Y., Robbins, T.W., 2006. Functions of frontostriatal systems in cognition: comparative neuropsychopharmacological studies in rats, monkeys and humans. Biol. Psychol. 73, 19-38.

Curtis, C.E., D'Esposito, M., 2003. Persistent activity in the prefrontal cortex during working memory. Trends Cogn. Sci. 7, 415-423.

Curtis, C.E., D'Esposito, M., 2006. Selection and maintenance of saccade goals in the human frontal eye fields. J. Neurophysiol. 95, 3923-3927.

Curtis, C.E., Rao, V.Y., D'Esposito, M., 2004. Maintenance of spatial and motor codes during oculomotor delayed response tasks. J. Neurosci. 24, 3944-3952.

Davachi, L., Wagner, A.D., 2002. Hippocampal contributions to episodic encoding: insights from relational and item-based learning. J. Neurophysiol. 88, 982-990.

Davachi, L., Mitchell, J.P., Wagner, A.D., 2003. Multiple routes to memory: distinct temporal lobe processes build item and source memories. Proc. Natl. Acad. Sci. U. S. A. 100, 2157-2162.

Dolan, R.J., Fletcher, P.C., 1997. Dissociating prefrontal and hippocampal function in episodic memory encoding. Nature 388, 582-585.

Eichenbaum, H., 2000. A cortical-hippocampal system for declarative memory. Nat. Rev., Neurosci. 1, 41-50.

Eichenbaum, H., 2004. Hippocampus: cognitive processes and neural representations that underlie declarative memory. Neuron 44, 109-120.

Fernández, G., Tendolkar, I., 2001. Integrated brain activity in medial temporal and prefrontal areas predicts subsequent memory performance: human declarative memory formation at the system level. Brain Res. Bull. 55, 1-9.

Fernández, G., Weyerts, H., Schrader-Bolsche, M., Tendolkar, I., Smid, H.G., Tempelmann, C., Hinrichs, H., Scheich, H., Elger, C.E., Mangun, G.R., Heinze, H.J., 1998. Successful verbal encoding into episodic memory engages the posterior hippocampus: a parametrically analyzed functional magnetic resonance imaging study. J. Neurosci. 18, 1841-1847.

Fletcher, P.C., Frith, C.D., Baker, S.C., Shallice, T., Frackowiak, R.S., Dolan, R.J., 1995a. The mind's eye-precuneus activation in memoryrelated imagery. NeuroImage 2, 195-200.

Fletcher, P.C., Frith, C.D., Grasby, P.M., Shallice, T., Frackowiak, R.S., Dolan, R.J., 1995b. Brain systems for encoding and retrieval of auditoryverbal memory: an in vivo study in humans. Brain 118, 401-416.

Fletcher, P.C., Shallice, T., Frith, C.D., Frackowiak, R.S., Dolan, R.J., 1996. Brain activity during memory retrieval: the influence of imagery and semantic cueing. Brain 119, 1587-1596.

Friston, K., Holmes, A.P., Worsley, K., Poline, J., Frackowiak, R., 1995. Statistical parametric maps in functional imaging: a general linear approach. Hum. Brain Mapp. 2, 189-210.

Fuster, J.M., Bodner, M., Kroger, J.K., 2000. Cross-modal and crosstemporal association in neurons of frontal cortex. Nature 405, 347-350.

Genovese, C.R., Lazar, N.A., Nichols, T., 2002. Thresholding of statistical maps in functional neuroimaging using the false discovery rate. NeuroImage 15, 870-878.
Hart, S.J., Davenport, M.L., Hooper, S.R., Belger, A., 2006. Visuospatial executive function in Turner syndrome: functional MRI and neurocognitive findings. Brain 129, 1125-1136.

Henke, K., Buck, A., Weber, B., Wieser, H.G., 1997. Human hippocampus establishes associations in memory. Hippocampus 7, 249-256.

Henke, K., Weber, B., Kneifel, S., Wieser, H.G., Buck, A., 1999. Human hippocampus associates information in memory. Proc. Natl. Acad. Sci. U. S. A. $96,5884-5889$.

Jackson III, O., Schacter, D.L., 2004. Encoding activity in anterior medial temporal lobe supports subsequent associative recognition. NeuroImage 21, 456-462.

Kao, Y.C., Davis, E.S., Gabrieli, J.D., 2005. Neutral correlates of actual and predicted memory formation. Nat. Neurosci. 8, 1776-1783.

Kirchhoff, B.A., Wagner, A.D., Maril, A., Stern, C.E., 2000. Prefrontal circuitry for episodic encoding and subsequent memory. J. Neurosci. 20, 6173-6180.

Kumaran, D., Maguire, E.A., 2006. The dynamics of hippocampal activation during encoding of overlapping sequences. Neuron 49, 617-629.

Levy, R., Friedman, H.R., Davachi, L., Goldman-Rakic, P.S., 1997. Differential activation of the caudate nucleus in primates performing spatial and nonspatial working memory tasks. J. Neurosci. 17, 3870-3882.

Luo, J., Niki, K., 2005. Does hippocampus associate discontiguous events? Evidence from event related fMRI. Hippocampus 15, 141-148.

Mather, M., Mitchell, K.J., Rave, C.L., Novak, D.L., Greene, E.J., Johnson, M.K., 2006. Emotional arousal can impair feature binding in working memory. J. Cogn. Neurosci. 18, 614-625.

Messinger, A., Squire, L.R., Zola, S.M., Albright, T.D., 2001. Neuronal representations of stimulus associations develop in the temporal lobe during learning. Proc. Natl. Acad. Sci. U. S. A. 98, 12239-12244.

Mitchell, J.P., Macrae, C.N., Banaji, M.R., 2004. Encoding-specific effects of social cognition on the neural correlates of subsequent memory. J. Neurosci. 24, 4912-4917.

Miyashita, Y., 2000. Visual associative long-term memory: encoding and retrieval in inferotemporal cortex of the primate. In: Gazzaniga, M.S. (Ed.), The New Cognitive Neurosciences. MIT, Cambridge, MA, pp. 379-392.

Miyashita, Y., 2004. Cognitive memory: cellular and network machineries and their top-down control. Science 306, 435-440.

Miyashita, Y., Hayashi, T., 2000. Neural representation of visual objects: encoding and top-down activation. Curr. Opin. Neurobiol. 10, 187-194.

Murray, E.A., Gaffan, D., Mishkin, M., 1993. Neural substrates of visual stimulus-stimulus association in rhesus monkeys. J. Neurosci. 13, 4549-4561.

Nagahama, Y., Okada, T., Katsumi, Y., Hayashi, T., Yamauchi, H., Sawamoto, N., et al., 1999. Transient neural activity in the medial superior frontal gyrus and precuneus time locked with attention shift between object features. NeuroImage 10, 193-199.

Narayanan, N.S., Prabhakaran, V., Bunge, S.A., Christoff, K., Fine, E.M., Gabrieli, J.D., 2005. The role of the prefrontal cortex in the maintenance of verbal working memory: an event-related fMRI analysis. Neuropsychology 19, 223-232.

Naya, Y., Sakai, K., Miyashita, Y., 1996. Activity of primate inferotemporal neurons related to a sought target in pair-association task. Proc. Natl. Acad. Sci. U. S. A. 93, 2664-2669.

Naya, Y., Yoshida, M., Miyashita, Y., 2001. Backward spreading of memoryretrieval signal in the primate temporal cortex. Science 291, 661-664.

O’Reilly, R.C., Frank, M.J., 2006. Making working memory work: a computational model of learning in the prefrontal cortex and basal ganglia. Neural Comput. 18, 283-328.

Otten, L.J., Henson, R.N., Rugg, M.D., 2001. Depth of processing effects on neural correlates of memory encoding: relationship between findings from across- and within-task comparisons. Brain 124, 399-412.

Paller, K.A., Wagner, A.D., 2002. Observing the transformation of experience into memory. Trends Cogn. Sci. 6, 93-102.

Pasupathy, A., Miller, E.K., 2005. Different time courses of learning-related activity in the prefrontal cortex and striatum. Nature 433, 873-876.

Petrides, M., 1985. Deficits on conditional associative-learning tasks after 
frontal- and temporal-lobe lesions in man. Neuropsychologia 23, 601-614.

Petrides, M., 1997. Visuo-motor conditional associative learning after frontal and temporal lesions in the human brain. Neuropsychologia 35, 989-997.

Prabhakaran, V., Narayanan, K., Zhao, Z., Gabrieli, J.D., 2000. Integration of diverse information in working memory within the frontal lobe. Nat. Neurosci. 3, 85-90.

Prince, S.E., Daselaar, S.M., Cabeza, R., 2005. Neural correlates of relational memory: successful encoding and retrieval of semantic and perceptual associations. J. Neurosci. 25, 1203-1210.

Ranganath, C., D'Esposito, M., 2005. Directing the mind's eye: prefrontal, inferior and medial temporal mechanisms for visual working memory. Curr. Opin. Neurobiol. 15, 175-182.

Ranganath, C., Yonelinas, A.P., Cohen, M.X., Dy, C.J., Tom, S.M., D'Esposito, M., 2004a. Dissociable correlates of recollection and familiarity within the medial temporal lobes. Neuropsychologia 42 , $2-13$.

Ranganath, C., Cohen, M.X., Brozinsky, C.J., 2004b. Inferior temporal, prefrontal, and hippocampal contributes to visual working memory maintenance and associative memory retrieval. J. Neurosci. 24, 3917-3925.

Ranganath, C., Heller, A., Cohen, M.X., Brozinsky, C.J., Rissman, J., 2005. Functional connectivity with the hippocampus during successful memory formation. Hippocampus 15, 997-1005.

Rawlins, J.N.P., 1985. Associations across time: the hippocampus as a temporary memory store. Behav. Brain Sci. 8, 479-496.

Rowe, J.B., Toni, I., Josephs, O., Frackowiak, R.S., Passingham, R.E., 2000. The prefrontal cortex: response selection or maintenance within working memory? Science 288, 1656-1660.

Sakai, K., 2003. Reactivation of memory: role of medial temporal lobe and prefrontal cortex. Rev. Neurosci. 14, 241-252.

Sakai, K., Passingham, R.E., 2004. Prefrontal selection and medial temporal lobe reactivation in retrieval of short-term verbal information. Cereb. Cortex 14, 914-921.

Sakai, K., Rowe, J.B., Passingham, R.E., 2002a. Parahippocampal reactivation signal at retrieval after interruption of rehearsal. J. Neurosci. $22,6315-6320$.

Sakai, K., Rowe, J.B., Passingham, R.E., 2002b. Active maintenance in prefrontal area 46 creates distractor-resistant memory. Nat. Neurosci. 5, 479-484.

Schacter, D.L., Wagner, A.D., 1999. Medial temporal lobe activations in fMRI and PET studies of episodic encoding and retrieval. Hippocampus 9, 7-24.

Schon, K., Hasselmo, M.E., Lopresti, M.L., Tricarico, M.D., Stern, C.E., 2004. Persistence of parahippocampal representation in the absence of stimulus input enhances long-term encoding: a functional magnetic imaging study of subsequent memory after a delayed match-to-sample task. J. Neurosci. 24, 11088-11097.

Schon, K., Atri, A., Hasselmo, M.E., Tricarico, M.D., LoPresti, M.L., Stern, C.E., 2005. Scopolamine reduces persistent activity related to long-term encoding in the parahippocampal gyrus during delayed matching in humans. J. Neurosci. 25, 9112-9123.

Sommer, T., Rose, M., Gläscher, J., Wolbers, T., Büchel, C., 2005a.
Dissociable contributions within the medial temporal lobe to encoding of object-location associations. Learn. Mem. 12, 343-351.

Sommer, T., Rose, M., Weiller, C., Buchel, C., 2005b. Contributions of occipital, parietal and parahippocampal cortex to encoding of objectlocation associations. Neuropsychologia 43, 732-743.

Sperling, R.A., Chua, E., Cocchiarella, A., Giovannetti, E.R., Poldrack, R., Schacter, D.L., Albert, M.S., 2003. Putting names to faces: successful encoding of associative memories activates the anterior hippocampal formation. NeuroImage 20, 1400-1410.

Squire, L.R., Zola-Morgan, S., 1991. The medial temporal lobe memory system. Science $253,1380-1386$.

Squire, L.R., Stark, C.E., Clark, R.E., 2004. The medial temporal lobe. Annu. Rev. Neurosci. 27, 279-306.

Thompson-Schill, S.L., D’Esposito, M., Aguirre, G.K., Farah, M.J., 1997. Role of left inferior prefrontal cortex in retrieval of semantic knowledge: a reevaluation. Proc. Natl. Acad. Sci. U. S. A. 94, 14792-14797.

Tomita, H., Ohbayashi, M., Nakahara, K., Hasegawa, I., Miyashita, Y., 1999. Top-down signal from prefrontal cortex in executive control of memory retrieval. Nature 401, 699-703.

Tulving, E., 1983. Elements of Episodic Memory. Oxford Univ. Press, New York.

Tulving, E., 2002. Episodic memory: from mind to brain. Annu. Rev. Psychol. 53, 1-25.

Uncapher, M.R., Otten, L.J., Rugg, M.D., 2006. Episodic encoding is more than the sum of its parts: an fMRI investigation of multifeatural contextual encoding. Neuron 52, 547-556.

Wagner, A.D., 1999. Working memory contributions to human learning and remembering. Neuron 22, 19-22.

Wagner, A.D., Schacter, D.L., Rotte, M., Koutstaal, W., Maril, A., Dale, A.M., Rosen, B.R., Buckner, R.L., 1998. Building memories: remembering and forgetting of verbal experiences as predicted by brain activity. Science $281,1188-1191$.

Wagner, A.D., Shannon, B.J., Kahn, I., Buchner, R.L., 2005. Parietal lobe contributions to episodic memory retrieval. Trends Cogn. Sci. 9, $445-453$.

Wallenstein, G.V., Eichenbaum, H., Hasselmo, M.E., 1998. The hippocampus as an associator of discontiguous events. Trends Neurosci. 21, $317-323$.

Wheeler, M.E., Buckner, R.L., 2003. Functional dissociation among components of remembering: control, perceived oldness, and content. J. Neurosci. 23, 3869-3880.

Wheeler, M.E., Petersen, S.E., Buckner, R.L., 2000. Memory's echo: vivid remembering reactivates sensory-specific cortex. Proc. Natl. Acad. Sci. U. S. A. $97,11125-11129$.

Wheeler, M.E., Shulman, G.L., Buckner, R.L., Miezin, F.M., Velanova, K., Petersen, S.E., 2006. Evidence for separate perceptual reactivation and search processes during remembering. Cereb. Cortex 16, 949-959.

Worsley, K.J., Marrett, S., Neelin, P., Vandal, A.C., Friston, K.J., Evans, A.C., 1996. A unified statistical approach for determining significant signals in images of cerebral activation. Hum. Brain Mapp. 4, 58-73.

Yonelinas, A.P., Hopfinger, J.B., Buonocore, M.H., Kroll, N.E., Baynes, K., 2001. Hippocampal, parahippocampal and occipital-temporal contributions to associative and item recognition memory: an fMRI study. NeuroReport 12, 359-363. 